\title{
The New Infinite Sequence Complexion Solutions of a Kind of Nonlinear Evolutionary Equations
}

\author{
Lina Yi, Jundong Bao, Taogetusang* \\ College of Mathematical Science, Inner Mongolia Normal University, Inner Mongolia, China \\ Email:573028703@qq.com,baojd@imnu.edu.cn, tgts@imnu.edu.cn
}

Received 2 November 2015; accepted 19 December 2015; published 22 December 2015

Copyright (C) 2015 by authors and Scientific Research Publishing Inc.

This work is licensed under the Creative Commons Attribution International License (CC BY). http://creativecommons.org/licenses/by/4.0/

(c) (i) Open Access

\begin{abstract}
The method combining the function transformation with the auxiliary equation is presented and the new infinite sequence complexion solutions of a class of nonlinear evolutionary equations are constructed. Step one, according to two function transformations, a class of nonlinear evolutionary equations is changed into two kinds of ordinary differential equations. Step two, using the first integral of the ordinary differential equations, two first order nonlinear ordinary differential equations are obtained. Step three, using function transformation, two first order nonlinear ordinary differential equations are changed to the ordinary differential equation that could be integrated. Step four, the new solutions, Bäcklund transformation and the nonlinear superposition formula of solutions of the ordinary differential equation that could be integrated are applied to construct the new infinite sequence complexion solutions of a class of nonlinear evolutionary equations. These solutions are consisting of two-soliton solutions, two-period solutions and solutions composed of soliton solutions and period solutions.
\end{abstract}

\section{Keywords}

Nonlinear Evolutionary Equation, Function Transformation, Nonlinear Superposition Formula of Solutions, Complexion New Solutions

\section{Introduction}

In Refs. [1]-[5], some methods were applied to research the following two nonlinear coupling systems, and a finite number of one soliton solutions were obtained.

*Corresponding author.

How to cite this paper: Yi, L.N., Bao, J.D. and Taogetusang (2015) The New Infinite Sequence Complexion Solutions of a Kind of Nonlinear Evolutionary Equations. Journal of Applied Mathematics and Physics, 3, 1624-1632. 


$$
\begin{gathered}
\sigma_{x x}=-\sigma+\sigma^{3}+l \sigma \rho^{2}, \\
\rho_{x x}=(h-l) \rho+m \rho^{3}+l \rho \sigma^{2}
\end{gathered}
$$

where $h, l$ and $m$ are constants; $\rho$ and $\sigma$ are scalars.

$$
\begin{gathered}
\sigma_{t t}-c^{2} \sigma_{x x}=a \sigma-b \sigma^{3}-b \sigma \rho^{2}, \\
\rho_{t t}-c^{2} \rho_{x x}=(a-4 e) \rho-b \rho^{3}-b \rho \sigma^{2}
\end{gathered}
$$

where $a, b, c^{2}$ and $e$ are constants; $\rho$ and $\sigma$ are scalars.

In Refs. [6]-[9], the new two-soliton solutions of the following a class of nonlinear evolutionary equations are constructed.

$$
\begin{gathered}
u_{x t}=u+\frac{1}{6}\left(u^{3}+3 u v^{2}\right)_{x x}, \quad v_{x t}=v+\frac{1}{6}\left(v^{3}+3 u^{2} v\right)_{x x} . \\
u_{x t}=u+\frac{1}{6}\left(u^{3}-3 u v^{2}\right)_{x x}, \quad v_{x t}=v-\frac{1}{6}\left(v^{3}-3 u^{2} v\right)_{x x} . \\
u_{x t}=u+\frac{1}{6}\left(u^{3}\right)_{x x}, \quad v_{x t}=v+\frac{1}{2}\left(u^{2} v\right)_{x x} . \\
u_{x t}=u+\frac{1}{6}\left(u^{3}+u v^{2}\right)_{x x}, \quad v_{x t}=v+\frac{1}{6}\left(v^{3}+u^{2} v\right)_{x x} . \\
u_{x t}=u+\frac{1}{6}\left(u^{3}\right)_{x x}, \quad v_{x t}=v+\frac{1}{6}\left(u^{2} v\right)_{x x} .
\end{gathered}
$$

In this paper, the method combining the function transformation with the auxiliary equation is applied to construct the new infinite sequence complexion solutions of a class of nonlinear evolutionary Equation (10) by the following steps.

Step one, in Part 2.1, according to function transformation (11), a class of nonlinear evolutionary Equation (10) is changed into two kinds of ordinary differential Equations (13), (14). And then with the help of the function transformation, ordinary differential Equations (13), (14) can be changed to ordinary differential Equations (19), (20). And the first integrals (21), (22) of the two ordinary differential equations are obtained. In Part 2.2, according to function transformation (12), a class of nonlinear evolutionary Equations (10) is changed into two kinds of ordinary differential Equations (25), (26). And then with the help of the function transformation, ordinary differential Equations (25), (26) can be changed to ordinary differential Equations (31), (32). And the first integrals (33), (34) of the two ordinary differential equations are obtained.

Step two, substituting the first integrals (21), (22) separately into the first equation of ordinary differential Equations (19), (20) yields two first order nonlinear ordinary differential Equations (23) and (24). Substituting the first integrals (33), (34) separately into the first equation of ordinary differential Equations (31), (32) yields two first order nonlinear ordinary differential Equations (35) and (36).

Step three, using function transformation, two first order nonlinear ordinary differential Equations (23) and (24) (or (23) and (24)) can be changed to the ordinary differential equation that could be integrated [10]-[13].

Step four, the new solutions, Bäcklund transformation and the nonlinear superposition formula of solutions of the ordinary differential equation that could be integrated [10]-[12] are applied to construct the new infinite sequence complexion solutions consisting of the Riemann $\theta$ function, the Jacobi elliptic function, the hyperbolic function, the trigonometric function and the rational function of a class of nonlinear evolutionary equations (10). These solutions are consisting of two-soliton solutions, two-period solutions and solutions composed of soliton solutions and period solutions.

$$
\left\{\begin{array}{l}
\delta_{1} u_{x x}+\alpha_{1} u_{t t}+\alpha_{2} u_{x t}=\alpha_{3} u+\alpha_{4} u^{3}+\alpha_{5} v^{2} u+\alpha_{6}\left(\gamma_{1} u^{3}+\alpha_{7} u v^{2}\right)_{x x} \\
\delta_{2} v_{x x}+\beta_{1} v_{t t}+\beta_{2} v_{x t}=\beta_{3} v+\beta_{4} v^{3}+\beta_{5} u^{2} v+\beta_{6}\left(\gamma_{2} v^{3}+\beta_{7} v u^{2}\right)_{x x}
\end{array} .\right.
$$

Here $\alpha_{j}, \beta_{i}(i=j=1,2, \cdots, 7), \gamma_{1}, \gamma_{2}, \delta_{1}, \delta_{2}$ are constants. 


\section{The Method Combining the Function Transformation with the Auxiliary Equation}

Assume the solutions of a class of nonlinear evolutionary Equation (10) as

$$
\begin{gathered}
\left\{\begin{array}{l}
u(x, t)=\frac{1}{2}\left[P_{1}\left(\xi_{1}\right)+Q_{1}\left(\eta_{1}\right)\right], \\
v(x, t)=\frac{1}{2}\left[P_{1}\left(\xi_{1}\right)-Q_{1}\left(\eta_{1}\right)\right] .
\end{array}\right. \\
\left\{\begin{array}{l}
u(x, t)=\frac{1}{2}\left[\exp \left[\int P_{2}\left(\xi_{2}\right) \mathrm{d} \xi_{2}\right]+\exp \left[\int Q_{2}\left(\eta_{2}\right) \mathrm{d} \eta_{2}\right]\right], \\
v(x, t)=\frac{1}{2}\left[\exp \left[\int P_{2}\left(\xi_{2}\right) \mathrm{d} \xi_{2}\right]-\exp \left[\int Q_{2}\left(\eta_{2}\right) \mathrm{d} \eta_{2}\right]\right],
\end{array}\right.
\end{gathered}
$$

where $\xi_{j}=\lambda_{j} x+\mu_{j} t, \eta_{j}=v_{j} x+\omega_{j} t, \lambda_{j}, \mu_{j}, v_{j}$ and $\omega_{j}$ are constants to be determined, and $\lambda_{j} \neq v_{j}$, $\mu_{j} \neq \omega_{j}(j=1,2)$.

\subsection{The First Kind of Function Transformation with the Two Kinds of Ordinary Differential Equations}

Substituting the first kind of function transformation (11) into a class of nonlinear evolutionary Equation (10) yields the following conclusions.

Case 1. When the coefficients of a class of nonlinear evolutionary equations satisfy the following conditions, the complexion solutions exist.

When $\alpha_{i}=\beta_{i}(i=1,2, \cdots, 7), \delta_{1}=\delta_{2}, \beta_{5}=3 \alpha_{4}, \beta_{7}=3 \gamma_{2}$ a class of nonlinear evolutionary Equation (10) has the complexion solutions, and the solutions are determined by the following nonlinear ordinary differential equations.

$$
\begin{aligned}
& \frac{\mathrm{d}^{2} P_{1}\left(\xi_{1}\right)}{\mathrm{d} \xi_{1}^{2}}=\frac{-3 \beta_{3} P_{1}^{4}\left(\xi_{1}\right)-36 \beta_{6} \lambda_{1}^{2} \gamma_{1}\left(P_{1}^{\prime}\left(\xi_{1}\right)\right)^{2}+P_{1}^{2}\left(\xi_{1}\right)\left[-\alpha_{5}+6\left(\lambda_{1}^{2} \delta_{2}+\beta_{1} \mu_{1}^{2}+\lambda_{1} \mu_{1} \alpha_{2}\right)\left(P_{1}^{\prime}\left(\xi_{1}\right)\right)^{2}\right]}{3\left[-3 \beta_{6} \gamma_{1} \lambda_{1}^{2}+\left(\beta_{1} \mu_{1}^{2}+\lambda_{1}^{2} \delta_{2}+\lambda_{1} \mu_{1} \alpha_{2}\right) P_{1}^{2}\left(\xi_{1}\right)\right] P_{1}\left(\xi_{1}\right)}, \\
& \frac{\mathrm{d}^{2} Q_{1}\left(\eta_{1}\right)}{\mathrm{d} \eta_{1}^{2}}=\frac{-3 \beta_{3} Q_{1}^{4}\left(\eta_{1}\right)-36 \beta_{6} v_{1}^{2} \gamma_{1}\left(Q_{1}^{\prime}\left(\eta_{1}\right)\right)^{2}+Q_{1}^{2}\left(\eta_{1}\right)\left[-\alpha_{5}+6\left(v_{1}^{2} \delta_{2}+\beta_{1} \omega_{1}^{2}+v_{1} \omega_{1} \alpha_{2}\right)\left(Q_{1}^{\prime}\left(\eta_{1}\right)\right)^{2}\right]}{3\left[-3 \beta_{6} \gamma_{1} v_{1}^{2}+\left(v_{1}^{2} \delta_{2}+v_{1} \alpha_{2} \omega_{1}+\beta_{1} \omega_{1}^{2}\right) Q_{1}^{2}\left(\eta_{1}\right)\right] Q_{1}\left(\eta_{1}\right)} .
\end{aligned}
$$

Case 2. The first integral of the second order nonlinear ordinary differential equations.

By the function transformation, the nonlinear ordinary differential Equations (13) and (14) are changed into the following two ordinary differential equations

$$
\begin{aligned}
& \left\{\begin{array}{l}
\frac{\mathrm{d} P_{1}}{\mathrm{~d} \xi_{1}}=Y_{1}, \\
\frac{\mathrm{d} Y_{1}}{\mathrm{~d} \xi_{1}}=\frac{-3 \beta_{3} P_{1}^{4}-\alpha_{5} P_{1}^{2}+\left[6\left(\lambda_{1}^{2} \delta_{2}+\beta_{1} \mu_{1}^{2}+\lambda_{1} \mu_{1} \alpha_{2}\right) P_{1}^{2}-36 \beta_{6} \lambda_{1}^{2} \gamma_{1}\right] Y_{1}^{2}}{3\left[-3 \beta_{6} \gamma_{1} \lambda_{1}^{2}+\left(\beta_{1} \mu_{1}^{2}+\lambda_{1}^{2} \delta_{2}+\lambda_{1} \mu_{1} \alpha_{2}\right) P_{1}^{2}\right] P_{1}},
\end{array}\right. \\
& \left\{\begin{array}{l}
\frac{\mathrm{d} Q_{1}}{\mathrm{~d} \eta_{1}}=X_{1}, \\
\frac{\mathrm{d} X_{1}}{\mathrm{~d} \eta_{1}}=\frac{-3 \beta_{3} Q_{1}^{4}-\alpha_{5} Q_{1}^{2}+\left[6\left(v_{1}^{2} \delta_{2}+\beta_{1} \omega_{1}^{2}+v_{1} \omega_{1} \alpha_{2}\right) Q_{1}^{2}-36 \beta_{6} v_{1}^{2} \gamma_{1}\right] X_{1}^{2}}{3\left[-3 \beta_{6} \gamma_{1} v_{1}^{2}+\left(v_{1}^{2} \delta_{2}+v_{1} \alpha_{2} \omega_{1}+\beta_{1} \omega_{1}^{2}\right) Q_{1}^{2}\right] Q_{1}} .
\end{array}\right.
\end{aligned}
$$

And by the following transformation (17) and (18), the two ordinary differential Equations (15) and (16) can be expressed by the form of (19) and (20).

$$
\mathrm{d} \xi_{1}=3\left[-3 \beta_{6} \gamma_{1} \lambda_{1}^{2}+\left(\beta_{1} \mu_{1}^{2}+\lambda_{1}^{2} \delta_{2}+\lambda_{1} \mu_{1} \alpha_{2}\right) P_{1}^{2}\right] \mathrm{d} \tau_{1}
$$




$$
\begin{aligned}
& \mathrm{d} \eta_{1}=3\left[-3 \beta_{6} \gamma_{1} v_{1}^{2}+\left(v_{1}^{2} \delta_{2}+v_{1} \alpha_{2} \omega_{1}+\beta_{1} \omega_{1}^{2}\right) Q_{1}^{2}\right] \mathrm{d} \zeta_{1} . \\
& \left\{\frac{\mathrm{d} P_{1}}{\mathrm{~d} \tau_{1}}=3 Y_{1}\left[-3 \beta_{6} \gamma_{1} \lambda_{1}^{2}+\left(\beta_{1} \mu_{1}^{2}+\lambda_{1}^{2} \delta_{2}+\lambda_{1} \mu_{1} \alpha_{2}\right) P_{1}^{2}\right]\right. \text {, } \\
& \frac{\mathrm{d} Y_{1}}{\mathrm{~d} \tau_{1}}=\frac{1}{P_{1}}\left[-3 \beta_{3} P_{1}^{4}-\alpha_{5} P_{1}^{2}+\left[6\left(\lambda_{1}^{2} \delta_{2}+\beta_{1} \mu_{1}^{2}+\lambda_{1} \mu_{1} \alpha_{2}\right) P_{1}^{2}-36 \beta_{6} \lambda_{1}^{2} \gamma_{1}\right] Y_{1}^{2}\right] \text {. } \\
& \left\{\begin{array}{l}
\frac{\mathrm{d} Q_{1}}{\mathrm{~d} \zeta_{1}}=3 X_{1}\left[-3 \beta_{6} \gamma_{1} v_{1}^{2}+\left(v_{1}^{2} \delta_{2}+v_{1} \alpha_{2} \omega_{1}+\beta_{1} \omega_{1}^{2}\right) Q_{1}^{2}\right], \\
\frac{\mathrm{d} X_{1}}{\mathrm{~d} \zeta_{1}}=\frac{1}{Q_{1}}\left[-3 \beta_{3} Q_{1}^{4}-\alpha_{5} Q_{1}^{2}+\left[6\left(v_{1}^{2} \delta_{2}+\beta_{1} \omega_{1}^{2}+v_{1} \omega_{1} \alpha_{2}\right) Q_{1}^{2}-36 \beta_{6} v_{1}^{2} \gamma_{1}\right] X_{1}^{2}\right] .
\end{array}\right.
\end{aligned}
$$

By calculating, the following first integral of the ordinary differential Equations (19) and (20) are obtained

$$
\begin{aligned}
& Y_{1}^{2}=\frac{6 c_{1} P_{1}^{8}+M_{1} P_{1}^{6}+M_{2} P_{1}^{4}-2 \alpha_{5} \beta_{6} \gamma_{1} \lambda_{1}^{2} P_{1}^{2}}{6\left(-3 \beta_{6} \gamma_{1} \lambda_{1}^{2}+\left[\delta_{2} \lambda_{1}^{2}+\mu_{1}\left(\alpha_{2} \lambda_{1}+\beta_{1} \mu_{1}\right)\right] P_{1}^{2}\right)^{2}}, \\
& X_{1}^{2}=\frac{6 c_{2} Q_{1}^{8}+N_{1} Q_{1}^{6}+N_{2} Q_{1}^{4}-2 \alpha_{5} \beta_{6} \gamma_{1} v_{1}^{2} Q_{1}^{2}}{6\left(-3 \beta_{6} \gamma_{1} v_{1}^{2}+\left[\delta_{2} v_{1}^{2}+\omega_{1}\left(\alpha_{2} v_{1}+\beta_{1} \omega_{1}\right)\right] Q_{1}^{2}\right)^{2}} .
\end{aligned}
$$

Substituting the first integral (21) and (22) severally into the first equation of the ordinary differential Equations (19) and (20) yields the following two ordinary differential equations

$$
\begin{aligned}
& \left(\frac{\mathrm{d} P_{1}}{\mathrm{~d} \tau_{1}}\right)^{2}=\frac{3}{2}\left(6 c_{1} P_{1}^{8}+M_{1} P_{1}^{6}+M_{2} P_{1}^{4}-2 \alpha_{5} \beta_{6} \gamma_{1} \lambda_{1}^{2} P_{1}^{2}\right), \\
& \left(\frac{\mathrm{d} Q_{1}}{\mathrm{~d} \zeta_{1}}\right)^{2}=\frac{3}{2}\left(6 c_{2} Q_{1}^{8}+N_{1} Q_{1}^{6}+N_{2} Q_{1}^{4}-2 \alpha_{5} \beta_{6} \gamma_{1} v_{1}^{2} Q_{1}^{2}\right),
\end{aligned}
$$

where $M_{1}=6 \beta_{3} \delta_{2} \lambda_{1}^{2}+6 \alpha_{2} \beta_{3} \lambda_{1} \mu_{1}+6 \beta_{1} \beta_{3} \mu_{1}^{2}, \quad M_{2}=-9 \beta_{3} \beta_{6} \gamma_{1} \lambda_{1}^{2}+\alpha_{5} \delta_{2} \lambda_{1}^{2}+\alpha_{2} \alpha_{5} \lambda_{1} \mu_{1}+\alpha_{5} \beta_{1} \mu_{1}^{2}$, $N_{1}=6 \beta_{3} \delta_{2} v_{1}^{2}+6 \alpha_{2} \beta_{3} v_{1} \omega_{1}+6 \beta_{1} \beta_{3} \omega_{1}^{2}, \quad N_{2}=-9 \beta_{3} \beta_{6} \gamma_{1} v_{1}^{2}+\alpha_{5} \delta_{2} v_{1}^{2}+\alpha_{2} \alpha_{5} v_{1} \omega_{1}+\alpha_{5} \beta_{1} \omega_{1}^{2}, \quad c_{1}$ and $c_{2}$ are arbitrary constants.

\subsection{The Second Kind of Function Transformation with the Two Kinds of Ordinary Differential Equations}

Substituting the second kind of function transformation (12) into a class of nonlinear evolutionary Equation (10) yields the following conclusions.

Case 1 . When the coefficients of a class of nonlinear evolutionary equations satisfy the following conditions, the complexion solutions exist.

When $\alpha_{i}=\beta_{i}(i=1,2, \cdots, 7), \delta_{1}=\delta_{2}, \beta_{5}=3 \alpha_{4}, \beta_{7}=3 \gamma_{2}$ a class of nonlinear evolutionary Equation (10) has the complexion solutions, and the solutions are determined by the following nonlinear ordinary differential equations.

$$
\begin{aligned}
\frac{\mathrm{d}^{2} P_{2}\left(\xi_{2}\right)}{\mathrm{d} \xi_{2}^{2}}=-\frac{3 \beta_{3}+\left[27 \beta_{6} \gamma_{1} \lambda_{2}^{2} P_{2}^{2}\left(\xi_{2}\right)+\alpha_{5}\right] \exp \left[2 \int P_{2}\left(\xi_{2}\right) \mathrm{d} \xi_{2}\right]-\left(3 \beta_{1} \mu_{2}^{2}+3 \lambda_{2} \mu_{2} \alpha_{2}+3 \lambda_{2}^{2} \delta_{2}\right) P_{2}^{2}\left(\xi_{2}\right)}{3\left(-\lambda_{2}^{2} \delta_{2}+3 \beta_{6} \gamma_{1} \lambda_{2}^{2} \exp \left[2 \int P_{2}\left(\xi_{2}\right) \mathrm{d} \xi_{2}\right]-\lambda_{2} \mu_{2} \alpha_{2}-\beta_{1} \mu_{2}^{2}\right)}, \\
\frac{\mathrm{d}^{2} Q_{2}\left(\eta_{2}\right)}{\mathrm{d} \eta_{2}^{2}}=-\frac{3 \beta_{3}+\left[27 \beta_{6} \gamma_{1} v_{2}^{2} Q_{2}^{2}\left(\eta_{2}\right)+\alpha_{5}\right] \exp \left[2 \int Q_{2}\left(\eta_{2}\right) \mathrm{d} \eta_{2}\right]-\left(3 \beta_{1} \omega_{2}^{2}+3 v_{2} \omega_{2} \alpha_{2}+3 v_{2}^{2} \delta_{2}\right) Q_{2}^{2}\left(\eta_{2}\right)}{3\left(-v_{2}^{2} \delta_{2}+3 \beta_{6} \gamma_{1} v_{2}^{2} \exp \left[2 \int Q_{2}\left(\eta_{2}\right) \mathrm{d} \eta_{2}\right]-\alpha_{2} \omega_{2} v_{2}-\beta_{1} \omega_{2}^{2}\right)} .
\end{aligned}
$$

The ordinary differential Equations (25) and (26) can be expressed by the following forms. 


$$
\begin{aligned}
& \left\{\begin{array}{l}
\frac{\mathrm{d} Y_{2}}{\mathrm{~d} \xi_{2}}=2 P_{2} Y_{2}, \\
\frac{\mathrm{d} P_{2}}{\mathrm{~d} \xi_{2}}=-\frac{3 \beta_{3}+\alpha_{5} Y_{2}-\left(3 \beta_{1} \mu_{2}^{2}+3 \lambda_{2} \mu_{2} \alpha_{2}+3 \lambda_{2}^{2} \delta_{2}-27 \beta_{6} \gamma_{1} \lambda_{2}^{2} Y_{2}\right) P_{2}^{2}}{3\left(-\lambda_{2}^{2} \delta_{2}+3 \beta_{6} \gamma_{1} \lambda_{2}^{2} Y_{2}-\lambda_{2} \mu_{2} \alpha_{2}-\beta_{1} \mu_{2}^{2}\right)}
\end{array}\right. \\
& \left\{\begin{array}{l}
\frac{\mathrm{d} X_{2}}{\mathrm{~d} \eta_{2}}=2 Q_{2} X_{2} \\
\frac{\mathrm{d} Q_{2}}{\mathrm{~d} \eta_{2}}=-\frac{3 \beta_{3}+\alpha_{5} X_{2}-\left(3 \beta_{1} \omega_{2}^{2}+3 v_{2} \omega_{2} \alpha_{2}+3 v_{2}^{2} \delta_{2}-27 \beta_{6} \gamma_{1} v_{2}^{2} X_{2}\right) Q_{2}^{2}}{3\left(-v_{2}^{2} \delta_{2}+3 \beta_{6} \gamma_{1} v_{2}^{2} X_{2}-\alpha_{2} \omega_{2} v_{2}-\beta_{1} \omega_{2}^{2}\right)} .
\end{array}\right.
\end{aligned}
$$

And by the following transformation (29) and (30), the two ordinary differential Equations (27), (28) can be expressed by the form of (31), (32).

$$
\begin{aligned}
& \mathrm{d} \xi_{2}=3\left(-\lambda_{2}^{2} \delta_{2}+3 \beta_{6} \gamma_{1} \lambda_{2}^{2} Y_{2}-\lambda_{2} \mu_{2} \alpha_{2}-\beta_{1} \mu_{2}^{2}\right) \mathrm{d} \tau_{2}, \\
& \mathrm{~d} \eta_{2}=3\left(-v_{2}^{2} \delta_{2}+3 \beta_{6} \gamma_{1} v_{2}^{2} X_{2}-\alpha_{2} \omega_{2} v_{2}-\beta_{1} \omega_{2}^{2}\right) \mathrm{d} \zeta_{2} \text {. } \\
& \int \frac{\mathrm{d} Y_{2}}{\mathrm{~d} \tau_{2}}=6 P_{2} Y_{2}\left(-\lambda_{2}^{2} \delta_{2}+3 \beta_{6} \gamma_{1} \lambda_{2}^{2} Y_{2}-\lambda_{2} \mu_{2} \alpha_{2}-\beta_{1} \mu_{2}^{2}\right), \\
& \frac{\mathrm{d} P_{2}}{\mathrm{~d} \tau_{2}}=-\left[3 \beta_{3}+\alpha_{5} Y_{2}-\left(3 \beta_{1} \mu_{2}^{2}+3 \lambda_{2} \mu_{2} \alpha_{2}+3 \lambda_{2}^{2} \delta_{2}-27 \beta_{6} \gamma_{1} \lambda_{2}^{2} Y_{2}\right) P_{2}^{2}\right] . \\
& \int \frac{\mathrm{d} X_{2}}{\mathrm{~d} \zeta_{2}}=6 Q_{2} X_{2}\left(-v_{2}^{2} \delta_{2}+3 \beta_{6} \gamma_{1} v_{2}^{2} X_{2}-\alpha_{2} \omega_{2} v_{2}-\beta_{1} \omega_{2}^{2}\right), \\
& \frac{\mathrm{d} Q_{2}}{\mathrm{~d} \zeta_{2}}=-\left[3 \beta_{3}+\alpha_{5} X_{2}-\left(3 \beta_{1} \omega_{2}^{2}+3 v_{2} \omega_{2} \alpha_{2}+3 v_{2}^{2} \delta_{2}-27 \beta_{6} \gamma_{1} v_{2}^{2} X_{2}\right) Q_{2}^{2}\right] .
\end{aligned}
$$

Case 2. The first integral of the second order nonlinear ordinary differential equations.

By calculating, the following first integral of the ordinary differential Equations (31), (32) are obtained.

$$
\begin{aligned}
P_{2}^{2} & =\frac{6 c_{3}-2 \alpha_{5} \beta_{6} \gamma_{1} \lambda_{2}^{2} Y_{2}^{3}+M_{3} Y_{2}^{2}+M_{4} Y_{2}}{6\left[-3 \beta_{6} \gamma_{1} \lambda_{2}^{2} Y_{2}+\delta_{2} \lambda_{2}^{2}+\mu_{2}\left(\alpha_{2} \lambda_{2}+\beta_{1} \mu_{2}\right)\right]^{2} Y_{2}}, \\
Q_{2}^{2} & =\frac{6 c_{4}-2 \alpha_{5} \beta_{6} \gamma_{1} v_{2}^{2} X_{2}^{3}+N_{3} X_{2}^{2}+N_{4} X_{2}}{6\left[-3 \beta_{6} \gamma_{1} v_{2}^{2} X_{2}+\delta_{2} v_{2}^{2}+\omega_{2}\left(\alpha_{2} v_{2}+\beta_{1} \omega_{2}\right)\right]^{2} X_{2}} .
\end{aligned}
$$

Substituting the first integral (33) and (34) severally into the first equation of the ordinary differential Equations (31) and (32) yields the following two nonlinear ordinary differential equations

$$
\begin{aligned}
& \left(\frac{\mathrm{d} Y_{2}}{\mathrm{~d} \tau_{2}}\right)^{2}=6\left[-2 \alpha_{5} \beta_{6} \gamma_{1} \lambda_{2}^{2} Y_{2}^{4}+M_{3} Y_{2}^{3}+M_{4} Y_{2}^{2}+6 c_{3} Y_{2}\right], \\
& \left(\frac{\mathrm{d} X_{2}}{\mathrm{~d} \zeta_{2}}\right)^{2}=6\left[-2 \alpha_{5} \beta_{6} \gamma_{1} v_{2}^{2} X_{2}^{4}+N_{3} X_{2}^{3}+N_{4} X_{2}^{2}+6 c_{4} X_{2}\right],
\end{aligned}
$$

where $M_{3}=-9 \beta_{3} \beta_{6} \gamma_{1} \lambda_{2}^{2}+\alpha_{5}\left(\delta_{2} \lambda_{2}^{2}+\alpha_{2} \lambda_{2} \mu_{2}+\beta_{1} \mu_{2}^{2}\right), \quad M_{4}=6 \beta_{3}\left[\delta_{2} \lambda_{2}^{2}+\mu_{2}\left(\alpha_{2} \lambda_{2}+\beta_{1} \mu_{2}\right)\right]$, $N_{3}=-9 \beta_{3} \beta_{6} \gamma_{1} v_{2}^{2}+\alpha_{5}\left(\delta_{2} v_{2}^{2}+\alpha_{2} v_{2} \omega_{2}+\beta_{1} \omega_{2}^{2}\right), \quad N_{4}=6 \beta_{3}\left[\delta_{2} v_{2}^{2}+\omega_{2}\left(\alpha_{2} v_{2}+\beta_{1} \omega_{2}\right)\right], \quad c_{3}$ and $c_{4}$ are arbitrary constants.

\section{The New Infinite Sequence Complexion Solutions of a Class of Nonlinear Evolutionary Equations}

In some cases, according to the ordinary differential Equations (23) and (24) (or (35) and (36)), the new infinite 
sequence complexion solutions of a class of nonlinear evolutionary Equation (10) are constructed.

\subsection{The New Infinite Sequence Complexion Solutions Consisting of the Hyperbolic Function, the Trigonometric Function and the Rational Function}

When $c_{1}=c_{2}=0, \alpha_{5} \beta_{6} \gamma_{1} \neq 0$, by the following superposition formula, the new infinite sequence complexion solutions consisting of the hyperbolic function, the trigonometric function and the rational function of a class of nonlinear evolutionary Equation (10) are obtained.

$$
\left\{\begin{array}{l}
u_{m n}(x, t)=\frac{1}{2}\left[\left(P_{1}\right)_{m}\left(\xi_{1}\right)+\left(Q_{1}\right)_{n}\left(\eta_{1}\right)\right] \quad(m=1,2, \cdots), \\
v_{m n}(x, t)=\frac{1}{2}\left[\left(P_{1}\right)_{m}\left(\xi_{1}\right)-\left(Q_{1}\right)_{n}\left(\eta_{1}\right)\right] \quad(n=1,2, \cdots), \\
\mathrm{d} \xi_{1}=3\left[-3 \beta_{6} \gamma_{1} \lambda_{1}^{2}+\left(\beta_{1} \mu_{1}^{2}+\lambda_{1}^{2} \delta_{2}+\lambda_{1} \mu_{1} \alpha_{2}\right) P_{1}^{2}\right] \mathrm{d} \tau_{1}, \\
\mathrm{~d} \eta_{1}=3\left[-3 \beta_{6} \gamma_{1} v_{1}^{2}+\left(v_{1}^{2} \delta_{2}+v_{1} \alpha_{2} \omega_{1}+\beta_{1} \omega_{1}^{2}\right) Q_{1}^{2}\right] \mathrm{d} \zeta_{1},
\end{array}\right.
$$

where $\left(P_{1}\right)_{m}\left(\xi_{1}\right)$ and $\left(Q_{1}\right)_{n}\left(\eta_{1}\right)$ are determined by the following superposition formula [10]-[13].

$$
\begin{aligned}
& \left(P_{1}\right)_{m}\left(\tau_{1}\right)= \pm \frac{\sqrt{-A_{1}+\left(V_{1}\right)_{m}^{2}\left(\tau_{1}\right)}}{\sqrt{B_{1}-2 \sqrt{C_{1}}\left(V_{1}\right)_{m}\left(\tau_{1}\right)}}(m=1,2, \cdots), \\
& \left\{\left(V_{1}\right)_{m}\left(\tau_{1}\right)=\frac{\left(-M_{0} \mp \sqrt{M_{0}^{2}-4 L_{0} N_{0}}\right) R_{1}+2\left(L_{0}-N_{0} R_{1}\right)\left(V_{1}\right)_{m-1}\left(\tau_{1}\right)+\left(M_{0} \mp \sqrt{M_{0}^{2}-4 L_{0} N_{0}}\right)\left(V_{1}\right)_{m-1}^{2}\left(\tau_{1}\right)}{2\left[L_{0}+M_{0}\left(V_{1}\right)_{m-1}\left(\tau_{1}\right)+N_{0}\left(V_{1}\right)_{m-1}^{2}\left(\tau_{1}\right)\right]},\right. \\
& \left(V_{1}\right)_{0}\left(\tau_{1}\right)=-\sqrt{-R_{1}} \tanh \left(\sqrt{-R_{1}} \tau_{1}\right) \quad\left(-R_{1}=A_{1}=-3 \alpha_{5} \beta_{6} \gamma_{1} \lambda_{1}^{2}<0\right) \text {, } \\
& B_{1}=\frac{3}{2}\left[-9 \beta_{3} \beta_{6} \gamma_{1} \lambda_{1}^{2}+\alpha_{5} \delta_{2} \lambda_{1}^{2}+\alpha_{2} \alpha_{5} \lambda_{1} \mu_{1}+\alpha_{5} \beta_{1} \mu_{1}^{2}\right], C_{1}=\frac{3}{2}\left[6 \beta_{3} \delta_{2} \lambda_{1}^{2}+6 \alpha_{2} \beta_{3} \lambda_{1} \mu_{1}+6 \beta_{1} \beta_{3} \mu_{1}^{2}\right] \text {. } \\
& \left(P_{1}\right)_{m}\left(\tau_{1}\right)= \pm \frac{\sqrt{-A_{1}+\left(V_{1}\right)_{m}^{2}\left(\tau_{1}\right)}}{\sqrt{B_{1}-2 \sqrt{C_{1}}\left(V_{1}\right)_{m}\left(\tau_{1}\right)}}(m=1,2, \cdots), \\
& \left\{\left(V_{1}\right)_{m}\left(\tau_{1}\right)=\frac{\left(-M_{0} \mp \sqrt{M_{0}^{2}-4 L_{0} N_{0}}\right) R_{1}+2\left(L_{0}-N_{0} R_{1}\right)\left(V_{1}\right)_{m-1}\left(\tau_{1}\right)+\left(M_{0} \mp \sqrt{M_{0}^{2}-4 L_{0} N_{0}}\right)\left(V_{1}\right)_{m-1}^{2}\left(\tau_{1}\right)}{2\left[L_{0}+M_{0}\left(V_{1}\right)_{m-1}\left(\tau_{1}\right)+N_{0}\left(V_{1}\right)_{m-1}^{2}\left(\tau_{1}\right)\right]},\right. \\
& \left(V_{1}\right)_{0}\left(\tau_{1}\right)=\sqrt{R_{2}} \tan \left(\sqrt{R_{2}} \tau_{1}\right) \quad\left(-R_{1}=A_{1}=-3 \alpha_{5} \beta_{6} \gamma_{1} \lambda_{1}^{2}>0\right) \text {, } \\
& B_{1}=\frac{3}{2}\left[-9 \beta_{3} \beta_{6} \gamma_{1} \lambda_{1}^{2}+\alpha_{5} \delta_{2} \lambda_{1}^{2}+\alpha_{2} \alpha_{5} \lambda_{1} \mu_{1}+\alpha_{5} \beta_{1} \mu_{1}^{2}\right], C_{1}=\frac{3}{2}\left[6 \beta_{3} \delta_{2} \lambda_{1}^{2}+6 \alpha_{2} \beta_{3} \lambda_{1} \mu_{1}+6 \beta_{1} \beta_{3} \mu_{1}^{2}\right] \text {. } \\
& \left(P_{1}\right)_{m}\left(\tau_{1}\right)= \pm \frac{\sqrt{-A_{1}+\left(V_{1}\right)_{m}^{2}\left(\tau_{1}\right)}}{\sqrt{B_{1}-2 \sqrt{C_{1}}\left(V_{1}\right)_{m}\left(\tau_{1}\right)}}(m=1,2, \cdots), \\
& \left\{\left(V_{1}\right)_{m}\left(\tau_{1}\right)=\frac{2 L_{0}\left(V_{1}\right)_{m-1}\left(\tau_{1}\right)+\left(M_{0} \mp \sqrt{M_{0}^{2}-4 L_{0} N_{0}}\right)\left(V_{1}\right)_{m-1}^{2}\left(\tau_{1}\right)}{2\left[L_{0}+M_{0}\left(V_{1}\right)_{m-1}\left(\tau_{1}\right)+N_{0}\left(V_{1}\right)_{m-1}^{2}\left(\tau_{1}\right)\right]} \quad(m=1,2, \cdots),\right. \\
& \left(V_{1}\right)_{0}\left(\tau_{1}\right)=-\frac{1}{\tau_{1}} \quad\left(-R_{1}=A_{1}=-3 \alpha_{5} \beta_{6} \gamma_{1} \lambda_{1}^{2}=0\right) \text {, } \\
& B_{1}=\frac{3}{2}\left[-9 \beta_{3} \beta_{6} \gamma_{1} \lambda_{1}^{2}+\alpha_{5} \delta_{2} \lambda_{1}^{2}+\alpha_{2} \alpha_{5} \lambda_{1} \mu_{1}+\alpha_{5} \beta_{1} \mu_{1}^{2}\right], C_{1}=\frac{3}{2}\left[6 \beta_{3} \delta_{2} \lambda_{1}^{2}+6 \alpha_{2} \beta_{3} \lambda_{1} \mu_{1}+6 \beta_{1} \beta_{3} \mu_{1}^{2}\right] .
\end{aligned}
$$




$$
\begin{aligned}
& \left\{\begin{array}{l}
\left(Q_{1}\right)_{n}\left(\zeta_{1}\right)= \pm \frac{\sqrt{-A_{2}+\left(V_{2}\right)_{n}^{2}\left(\zeta_{1}\right)}}{\sqrt{B_{2}-2 \sqrt{C_{2}}\left(V_{2}\right)_{n}\left(\zeta_{1}\right)}}(n=1,2, \cdots), \\
\left(V_{2}\right)_{n}\left(\zeta_{1}\right)=\frac{\left(-M_{0} \mp \sqrt{M_{0}^{2}-4 L_{0} N_{0}}\right) R_{2}+2\left(L_{0}-N_{0} R_{2}\right)\left(V_{2}\right)_{n-1}\left(\zeta_{1}\right)+\left(M_{0} \mp \sqrt{M_{0}^{2}-4 L_{0} N_{0}}\right)\left(V_{2}\right)_{n-1}^{2}\left(\zeta_{1}\right)}{2\left[L_{0}+M_{0}\left(V_{2}\right)_{n-1}\left(\zeta_{1}\right)+N_{0}\left(V_{2}\right)_{n-1}^{2}\left(\zeta_{1}\right)\right]} \\
\left(V_{2}\right)_{0}\left(\zeta_{1}\right)=-\sqrt{-R_{2}} \tanh \left(\sqrt{-R_{2}} \zeta_{1}\right) \quad\left(-R_{2}=A_{2}=-3 \alpha_{5} \beta_{6} \gamma_{1} v_{1}^{2}<0\right), \\
B_{2}=\frac{3}{2}\left[-9 \beta_{3} \beta_{6} \gamma_{1} v_{1}^{2}+\alpha_{5} \delta_{2} v_{1}^{2}+\alpha_{2} \alpha_{5} v_{1} \omega_{1}+\alpha_{5} \beta_{1} \omega_{1}^{2}\right], C_{2}=\frac{3}{2}\left[6 \beta_{3} \delta_{2} v_{1}^{2}+6 \alpha_{2} \beta_{3} v_{1} \omega_{1}+6 \beta_{1} \beta_{3} \omega_{1}^{2}\right] . \\
\left(Q_{1}\right)_{n}\left(\zeta_{1}\right)= \pm \frac{\sqrt{-A_{2}+\left(V_{2}\right)_{n}^{2}\left(\zeta_{1}\right)}}{\sqrt{B_{2}-2 \sqrt{C_{2}}\left(V_{2}\right)_{n}\left(\zeta_{1}\right)}}(n=1,2, \cdots), \\
\left(V_{2}\right)_{n}\left(\zeta_{1}\right)=\frac{\left(-M_{0} \mp \sqrt{M_{0}^{2}-4 L_{0} N_{0}}\right) R_{2}+2\left(L_{0}-N_{0} R_{2}\right)\left(V_{2}\right)_{n-1}\left(\zeta_{1}\right)+\left(M_{0} \mp \sqrt{M_{0}^{2}-4 L_{0} N_{0}}\right)\left(V_{2}\right)_{n-1}^{2}\left(\zeta_{1}\right)}{2\left[L_{0}+M_{0}\left(V_{2}\right)_{n-1}\left(\zeta_{1}\right)+N_{0}\left(V_{2}\right)_{n-1}^{2}\left(\zeta_{1}\right)\right]} \\
\left(V_{2}\right)_{0}\left(\zeta_{1}\right)=\sqrt{R_{2}} \tan \left(\sqrt{R_{2}} \zeta_{1}\right)\left(-R_{2}=A_{2}=-3 \alpha_{5} \beta_{6} \gamma_{1} v_{1}^{2}>0\right), \\
B_{2}=\frac{3}{2}\left[-9 \beta_{3} \beta_{6} \gamma_{1} v_{1}^{2}+\alpha_{5} \delta_{2} v_{1}^{2}+\alpha_{2} \alpha_{5} v_{1} \omega_{1}+\alpha_{5} \beta_{1} \omega_{1}^{2}\right], C_{2}=\frac{3}{2}\left[6 \beta_{3} \delta_{2} v_{1}^{2}+6 \alpha_{2} \beta_{3} v_{1} \omega_{1}+6 \beta_{1} \beta_{3} \omega_{1}^{2}\right] . \\
B_{2}=\frac{3}{2}\left[-9 \beta_{3} \beta_{6} \gamma_{1} v_{1}^{2}+\alpha_{5} \delta_{2} v_{1}^{2}+\alpha_{2} \alpha_{5} v_{1} \omega_{1}+\alpha_{5} \beta_{1} \omega_{1}^{2}\right], C_{2}=\frac{3}{2}\left[6 \beta_{3} \delta_{2} v_{1}^{2}+6 \alpha_{2} \beta_{3} v_{1} \omega_{1}+6 \beta_{1} \beta_{3} \omega_{1}^{2}\right] . \\
\left(V_{1}\right)_{n}\left(\zeta_{1}\right)= \pm \frac{2 L_{0}\left(V_{2}\right)_{n-1}\left(\zeta_{1}\right)+\left(M_{0}+\left(V_{2}\right)_{n}^{2}\left(\zeta_{1}\right)\right.}{\sqrt{B_{2}-2 \sqrt{C_{2}}\left(V_{2}\right)_{n}\left(\zeta_{1}\right)}}(n=1,2, \cdots), \\
\left(\begin{array}{l}
\left.\zeta_{1}^{2}-4 L_{0} N_{0}\right)\left(V_{2}\right)_{n-1}^{2}\left(\zeta_{1}\right) \\
\left.\left(\zeta_{1}\right)+N_{0}\left(V_{2}\right)_{n-1}^{2}\left(\zeta_{1}\right)\right]
\end{array}\right.
\end{array}\right.
\end{aligned}
$$

In (38)-(43), $L_{0}, M_{0}$ and $N_{0}$ are arbitrary constants not all equal to zero.

Case 1. The hyperbolic function type new infinite sequence complexion two-soliton solutions of a class of nonlinear evolutionary Equation (10).

Substituting the solution determined by the superposition formula (38), (41) into (37) yields the hyperbolic function type new infinite sequence complexion two-soliton solutions.

Case 2. The new infinite sequence complexion solutions consisting of the hyperbolic function and the trigonometric function of a class of nonlinear evolutionary Equation (10).

Substituting the solution determined by the superposition formula (38), (42) into (37) yields the new infinite sequence complexion solutions composed of soliton solutions and the period solutions.

Case 3. The new infinite sequence complexion solutions consisting of the hyperbolic function and the rational function of a class of nonlinear evolutionary Equation (10).

Substituting the solution determined by the superposition formula (38) and (43) into (37) yields the new infinite sequence complexion solutions consisting of the hyperbolic function and the rational function.

Case 4. The trigonometric function type new infinite sequence two-period solutions of a class of nonlinear evolutionary Equation (10).

Substituting the solution determined by the superposition formula (39), (42) into (37) yields the trigonometric function type new infinite sequence two-period solutions. 
Case 5. The new infinite sequence complexion solutions consisting of the trigonometric function and the rational function of a class of nonlinear evolutionary Equation (10).

Substituting the solution determined by the superposition formula (39), (43) into (37) yields the new infinite sequence complexion solutions consisting of the trigonometric function and the rational function.

Case 6. The rational function type new infinite sequence solutions of a class of nonlinear evolutionary Equation (10).

Substituting the solution determined by the superposition formula (40) and (43) into (37) yields the rational function type new infinite sequence solutions.

When $c_{3}=c_{4}=0$, with the help of the ordinary differential Equations (35) and (36), the new infinite sequence complexion two-soliton and two-periods solutions consisting of the hyperbolic function, the trigonometric function and the rational function of a class of nonlinear evolutionary Equation (10) can also be obtained (not given here).

\subsection{The New Infinite Sequence Complexion Solutions Consisting of the Riemann Function, the Jacobi Elliptic Function, the Hyperbolic Function, the Trigonometric Function and the Rational Function}

With the help of the relative conclusions of the second kind of elliptic equation [11]-[13], by analyzing, the infinite sequence solutions of the ordinary differential Equations (35) and (36) are obtained. Then substituting these solutions into function transformation (44) yields the new infinite sequence complexion solutions consisting of the Riemann $\theta$ function, the Jacobi elliptic function, the hyperbolic function, the trigonometric function and the rational function of a class of nonlinear evolutionary Equation (10) in some kinds of cases. These solutions are consisting of two-soliton solutions, two-period solutions and solutions composed of soliton solutions and period solutions (not given concretely here).

$$
\left\{\begin{array}{l}
u_{m n}(x, t)=\frac{1}{2}\left[\exp \left[\int\left(P_{2}\right)_{m}\left(\xi_{2}\right) \mathrm{d} \xi_{2}\right]+\exp \left[\int\left(Q_{2}\right)_{n}\left(\eta_{2}\right) \mathrm{d} \eta_{2}\right]\right] \quad(m, n=1,2, \cdots), \\
v_{m n}(x, t)=\frac{1}{2}\left[\exp \left[\int\left(P_{2}\right)_{m}\left(\xi_{2}\right) \mathrm{d} \xi_{2}\right]-\exp \left[\int\left(Q_{2}\right)_{n}\left(\eta_{2}\right) \mathrm{d} \eta_{2}\right]\right] \quad(m, n=1,2, \cdots), \\
\mathrm{d} \xi_{2}=3\left(-\lambda_{2}^{2} \delta_{2}+3 \beta_{6} \gamma_{1} \lambda_{2}^{2} Y_{2}-\lambda_{2} \mu_{2} \alpha_{2}-\beta_{1} \mu_{2}^{2}\right) \mathrm{d} \tau_{2}, \\
\mathrm{~d} \eta_{2}=3\left(-v_{2}^{2} \delta_{2}+3 \beta_{6} \gamma_{1} v_{2}^{2} X_{2}-\alpha_{2} \omega_{2} \nu_{2}-\beta_{1} \omega_{2}^{2}\right) \mathrm{d} \zeta_{2} .
\end{array}\right.
$$

Case 1. When $\gamma_{1}=0, \alpha_{5} \beta_{6} \neq 0, c_{3} c_{4} \neq 0$, the new infinite sequence complexion solutions consisting of the Riemann $\theta$ function, the Jacobi elliptic function, the hyperbolic function, the trigonometric function and the rational function of a class of nonlinear evolutionary Equation (10) are obtained.

Case 2. When $\beta_{6}=0, \alpha_{5} \gamma_{1} \neq 0, c_{3} c_{4} \neq 0$, the new infinite sequence complexion solutions consisting of the Riemann $\theta$ function, the Jacobi elliptic function, the hyperbolic function, the trigonometric function and the rational function of a class of nonlinear evolutionary Equation (10) are obtained.

Case 3. When $\alpha_{5}=0, \beta_{6} \gamma_{1} \neq 0, c_{3} c_{4} \neq 0$, the new infinite sequence complexion solutions consisting of the Riemann $\theta$ function, the Jacobi elliptic function, the hyperbolic function, the trigonometric function and the rational function of a class of nonlinear evolutionary Equation (10) are obtained.

\section{Conclusions}

In Refs. [1]-[5], a finite number of one function type one soliton new solutions consisting of the Jacobi elliptic function, the hyperbolic function, the trigonometric function and the rational function of the nonlinear evolutionary Equations (1)-(4) were constructed. In Refs. [6]-[9], a finite number of complexion function type twosoliton new solutions consisting of the Jacobi elliptic function, the hyperbolic function, the trigonometric function and the rational function of the nonlinear evolutionary Equation (5) were constructed.

In this paper, the method combining the function transformation with the auxiliary equation is presented, and the problem of solving the solutions of a class of nonlinear evolutionary Equation (10) concluding the nonlinear evolutionary Equations (1)-(9) is considered; the new infinite sequence complexion solutions consisting of the Riemann $\theta$ function, the Jacobi elliptic function, the hyperbolic function, the trigonometric function and the 
rational function are obtained. These solutions are consisting of two-soliton solutions, two-period solutions and solutions composed of soliton solutions and period solutions.

When the coefficients of nonlinear evolutionary Equations (1) are $\alpha_{3}=\beta_{3}=h-l=-1, \alpha_{4}=\beta_{4}=m=1$, then they satisfy the condition $\alpha_{i}=\beta_{i}(i=1,2, \cdots, 7), \delta_{1}=\delta_{2}, \beta_{5}=3 \alpha_{4}, \beta_{7}=3 \gamma_{2}$ that the new infinite sequence complexion solutions exist. So, according to the method given in the Part 3 , the new infinite sequence complexion two-soliton and two-periods and so on solutions of nonlinear evolutionary Equation (1) are constructed.

When the coefficients of a class of nonlinear evolutionary Equation (10) are

$\delta_{1}=\delta_{2}=\alpha_{1}=\beta_{1}=\alpha_{4}=\alpha_{5}=\beta_{4}=\beta_{5}=0, \quad \alpha_{2}=\beta_{2}=\alpha_{3}=\beta_{3}=\gamma_{1}=\gamma_{2}=1, \alpha_{6}=\beta_{6}=\frac{1}{6}, \alpha_{7}=\beta_{7}=3$, it satisfies the condition that the new infinite sequence complexion solutions exist. Then according to the conclusions given in Part 3 in the paper, the new infinite sequence complexion two-soliton solutions, two-period solutions and solutions composed of soliton solutions and period solutions of nonlinear evolutionary Equation (5) are constructed.

\section{Acknowledgements}

Project supported by the Natural Science Foundation of China (Grant No. 11361040), the Science Research Foundation of Institution of Higher Education of Inner Mongolia Autonomous Region, China (Grant No. NJZY12031) and the Natural Science Foundation of Inner Mongolia Autonomous Region, China (Grant No. 2015MS0128).

\section{References}

[1] Wang, X.Y. and Zhao, N. (1991) New Solitons in Coupled Scalar Field Theory. Acta Physica Sinica, 40, 359-364.

[2] Fan, E.G., Zhang, H.Q. and Lin, G. (1998) Exact Solutions to the Nonlinear Coupled Scalar Field Equations. Acta Physica Sinica, 47, 1064-1070.

[3] Liu, C.P. (2000) The Soliton Solutions for a Class of Nonlinear Coupled Equations. Acta Physica Sinica, 49, 19041908.

[4] Li, D.S. and Zhang, H.Q. (2003) The New Doubly-Periodic Solutions for Nonlinear Coupled Scalar Field Equations (1). Acta Physica Sinica, 52, 2373-2378.

[5] Li, D.S. and Zhang, H.Q. (2003) The New Doubly-Periodic Solutions for Nonlinear Coupled Scalar Field Equations (2). Acta Physica Sinica, 52, 2379-2385.

[6] Pietrzyk, M., Kanattškov, I. and Bandelow, U. (2008) On the Propagation of Vector Ultra-Short Pulses. Journal of Nonlinear Mathematical Physics, 15, 70-162. http://dx.doi.org/10.2991/jnmp.2008.15.2.4

[7] Sakovich, S. (2008) Integrability of the Vector Short Pulse Equation. Journal of the Physical Society of Japan, 77, Article ID: 123001. http://dx.doi.org/10.1143/jpsj.77.123001

[8] Kozlov, S.A. and Sazonov, S.V. (1997) Nonlinear Propagation of Optical Pulses of a Few Oscillations Duration in Dielectric Media. Journal of Experimental and Theoretical Physics, 84, 221-228. http://dx.doi.org/10.1134/1.558109

[9] Rui, W.G. (2013) Different Kinds of Exact Solutions with Two-Loop Character of the Two-Component Short Pulse Equations of the First Kind. Communications in Nonlinear Science and Numerical Simulation, 18, 2667-2678. http://dx.doi.org/10.1016/j.cnsns.2013.01.020

[10] Taogetusang and Yi, L.N. (2014) New Complexion Two-Soliton Solutions to a Kind of Nonlinear Coupled System. Acta Physica Sinica, 63, Article ID: 160201.

[11] Taogetusang and Yi, L.N. (2014) New Infinite Sequence Solutions to Equations of Sine-Gordon Type. Acta Physica Sinica, 63, Article ID: 215202.

[12] Taogetusang, S. and Li, S.M. (2010) New Application to Riccati Equation. Chinese Physics B, 19, Article ID: 080303. http://dx.doi.org/10.1088/1674-1056/19/8/080303

[13] Wang, J.M. (2012) Riemann $\theta$ Function Solutions to Modified Korteweg de Vries-Sine-Gordon Equation. Acta Physica Sinica, 61, Article ID: 080201. 